\title{
ANÁLISIS MORFOMÉTRICO DE LA CASTA OBRERA DE Cortaritermes fulviceps (ISOPTERA, TERMITIDAE)
}

\author{
Giovana Annoni ${ }^{1}$, Juan Manuel Coronel ${ }^{1}$, Enrique Rafael Laffont ${ }^{1}$ \\ ${ }^{1}$ Laboratorio de Biología de los Invertebrados. Universidad Nacional del Nordeste. Facultad de Ciencias Exactas y Naturales y \\ Agrimensura. Av. Libertad 5470. CP 3400. Corrientes, Argentina. E-mail: erl@exa.unne.edu.ar
}

\section{RESUMEN}

Se procedió al análisis de las características morfométricas de la casta obrera de Cortaritermes fulviceps. Se midieron un total de 50 obreras mayores y 50 menores de cinco colonias (en cada colonia 10 individuos de cada categoría) procedentes del Parque Nacional Mburucuyá (Corrientes, Argentina). Los caracteres considerados fueron la longitud total del cuerpo, ancho máximo de la cabeza, longitud de la cápsula cefálica, ancho y longitud media del pronoto y longitud de la tibia posterior. Se registraron diferencias significativas en todos los caracteres entre obreras mayores y menores, solo la longitud de la cápsula cefálica presentó diferencias significativas entre obreras procedentes de diferentes colonias. Los caracteres del pronoto y la tibia fueron los más variables en las obreras mayores, mientras que en las menores fueron los relacionados a la cabeza.

Palabras claves: termites, análisis morfométrico, casta obrera

\section{ANÁLISE MORFOMÉTRICA DE OPERÁRIAS DE Cortaritermes fulviceps (ISOPTERA, TERMITIDAE)}

\section{RESUMO}

Analisaram-se as características morfométricas da casta operária de Cortaritermes fulviceps. Foram medidas um total de 50 operárias grandes e 50 pequenas de cinco colônias (em colônia 10 indivíduos de cada categoria) do Parque Nacional Mburucuyá (Corrientes, Argentina). Os caracteres considerados foram: comprimento total do corpo, largura máxima da cabeça, comprimento da cápsula cefálica, largura e comprimento médio do pronoto e comprimento da tíbia posterior. Houve diferenças significativas em todos os caracteres entre operárias grandes e pequenas. Apenas o comprimento da cápsula cefálica mostrou diferenças significativas entre operárias de diferentes colônias. Os caracteres do pronoto e da tíbia foram os que mais variaram dentre as operárias grandes, enquanto que nas operárias pequenas foram aqueles relacionados a cabeça.

Palavras-chave: cupins, análise morfométrica, casta operária

\section{INTRODUCCIÓN}

Uno de los rasgos más distintivos de los insectos sociales es la organización de sus colonias en un sistema de castas diferenciadas morfológica y funcionalmente.
En las sociedades de termites las obreras son las encargadas de realizar las actividades relacionadas con la obtención del alimento, construcción del nido, cuidados de las crías y otras castas, y también participan de la defensa de la colonia. Estas actividades 
pueden variar de acuerdo al tamaño y edad de las obreras, es por ello que el conocimiento de las proporciones de cada casta y su función en la colonia resultan fundamentales para entender la dinámica ecológica de una especie (Thorne, 1985).

La especie Cortaritermes fulviceps se encuentra distribuida en Argentina, Brazil, Paraguay y Uruguay (Constantino, 1998), en Argentina presenta una amplia distribución abarcando las regiones Paranaense, Chaqueña, del Espinal, Pampeana y del Monte (Torales et al., 2005; Torales et al., 2008).

Diversos estudios han demostrado que en especies ampliamente distribuidas (como en el caso de $C$. fulviceps) existen importantes variaciones morfométricas entre individuos que proceden de diferentes localidades, que se suman a las variaciones propias de cada colonia (Roonwal \& Sangal, 1957; Hostettler et al., 1995; Coronel \& Porcel, 2001, Coronel \& Porcel, 2002; Manzoor \& Akhtar, 2006).

C. fulviceps presenta obreras dimórficas (obreras mayores y menores), sin embargo las características morfométricas y rangos de variaciones de cada grupo, que permitan una clara diferenciación entre estos dos tipos de obreras, no han sido establecidos hasta el momento. Esta información resulta de gran importancia al momento de realizar estudios sobre la dinámica poblacional y composición cuantitativa de las colonias ya que las proporciones de obreras mayores y menores pueden variar, dentro de una misma especie, según la edad de las colonias, época del año (Darlington et al., 1992) o cantidad de reinas presentes en el nido (Thorne, 1985).

En otras especies de Termitidae se ha demostrado que cada grupo puede realizar tareas diferentes en lo que respecta al funcionamiento de la colonia (Hinze et al., 2002), de modo tal que las variaciones en las proporciones de ambos grupos de obreras puede afectar la biología de todo el termitero.

Con el objeto de llenar este vacío existente en el conocimiento de la especie, se consideró oportuno realizar el estudio morfométrico de la casta obrera en $C$. fulviceps.

\section{MATERIALES Y MÉTODOS}

Para este estudio se utilizaron muestras de $C$. fulviceps depositadas en la Colección de Isópteros de la Facultad de Ciencias Exactas y Naturales y Agrimensura (Universidad Nacional del Nordeste, Corrientes, Argentina), ubicada en el Laboratorio 6 de Biología de los Invertebrados e identificada con el acrónimo "FACENAC", esta colección (la mas grande del orden Isoptera en la Argentina) alberga mas de 2500 muestras de termites no solo de Argentina sino también de otros países sudamericanos y europeos.

Para las mediciones de los caracteres morfométricos se siguieron los criterios establecidos por Roonwal, 1969 y las variables consideradas fueron las siguientes: Longitud total del cuerpo (LTC): distancia entre el extremo anterior de la cabeza y el extremo posterior del cuerpo, medida en vista dorsal.

Ancho máximo de la cabeza (AMC): distancia entre dos paralelas que marcan el margen mas externo de la cápsula cefálica (incluye los ojos compuestos cuando están presentes), medida en vista dorsal.

Longitud de la cápsula cefálica (LCC): Distancia entre dos paralelas que marcan el margen posterior de la cápsula cefálica y la articulación externa de la mandíbula izquierda, medida en vista dorsal.

Ancho máximo del pronoto (AMP): Distancia entre dos paralelas que marcan los márgenes laterales del pronoto, en su punto de máxima separación, medida en vista dorsal. 
Longitud media del pronoto (LMP): Distancia entre dos paralelas que marcan el borde anterior y posterior del pronoto a lo largo de la línea media longitudinal, medida en vista dorsal.

Longitud de la tibia posterior (LTP): Distancia entre dos paralelas que marcan los márgenes proximal y distal de la tibia del tercer par de piernas, excluyendo las espinas apicales, medida con la tibia mantenida en posición horizontal.

Para efectuar las dimensiones en las obreras, estas fueron colocadas individualmente en una cápsula de Petri conteniendo arena blanca fina y cubiertas con alcohol $70 \%$. Las mediciones se realizaron con ocular micrométrico adosado a un microscopio estereoscópico Olympus SZ 70H, también se registró el número de segmentos de la antena izquierda.

Las mediciones se realizaron en 5 muestras (FACENAC 0891; FACENAC 0892; FACENAC 0899; FACENAC 0992 y FACENAC 1284) todas procedentes del Parque Nacional Mburucuyá (Corrientes, Argentina), en cada una de estas muestras se midieron 10 obreras mayores y 10 menores.

Con los datos obtenidos se calcularon los valores promedios, máximos y mínimos, desvío estándar y coeficiente de variación de cada variable, se midió además la correlación entre todos los caracteres considerados con el coeficiente de correlación $r$ de Pearson, se analizaron también las diferencias entre obreras mayores y menores y entre obreras de diferentes muestras mediante el análisis de la varianza no paramétrico de Kruskal Wallis. Se aplicó la técnica de análisis de componentes principales sobre la matriz de datos previamente estandarizada.

\section{RESULTADOS Y DISCUSIÓN}

En Cortaritermes fulviceps, el número de segmentos antenales en la casta obrera varía entre 11 y 15, las obreras mayores presentaron antenas con $13(\mathrm{n}=21), 14(\mathrm{n}=$ 25) y $15(n=4)$ segmentos, mientras que en las menores se observaron individuos con 11 $(\mathrm{n}=16), 12(\mathrm{~N}=24)$ y $13(\mathrm{n}=10)$ segmentos. Esto permite suponer que en $C$. fulviceps, tal como ocurre en el género Nasutitermes (Roisin, 2000, Buschini y Costa Leonardo, 2002) la casta obrera estaría formada por dos estadíos, ya que en las termites, como en otros insectos hemimetábolos, el número de antenitos se incrementa con cada muda (Noirot, 1955).

En la Tabla 1 se presenta la estadística descriptiva de los caracteres analizados. Puede observarse que, excepto LTC, hay solapamiento en todos los restantes caracteres; también que los caracteres cefálicos son mas variables en las obreras menores, mientras que las dimensiones del pronoto y tibia varían mas en las obreras mayores.

En cuanto a las correlaciones entre caracteres pudo observarse que en las obreras mayores las correlaciones mas altas se dan entre LTC y AMP, mientras que en las menores ocurren entre AMC y LCC, en las obreras mayores LMP presentó correlaciones positivas y significativas con todos los demás caracteres, excepto LTP, mientras que en las menores solo lo hizo con AMC y AMP (Tabla2).

Al analizar las diferencias entre obreras mayores y menores mediante el análisis de la varianza de Kruskal-Wallis se encontraron diferencias significativas en todos los caracteres considerados, mientras que al comparar las obreras procedentes de las diferentes muestras solo se observaron diferencias significativas en LCC (Tabla 3).

Al analizar las diferencias entre obreras mayores y menores mediante el análisis de la varianza de Kruskal-Wallis se encontraron diferencias significativas en todos los caracteres considerados, mientras que al comparar las obreras procedentes de las diferentes muestras solo se observaron diferencias significactivas en LCC (Tabla 3) 
Tabla 1. Estadística descriptiva de los caracteres morfométricos analizados en obreras de $C$. fulviceps $. \mathrm{P}=$ Promedio $; \mathrm{DE}=$ Desvío Estándar; Min = Mínimo; Max = Máximo.

\begin{tabular}{ccccccccccc}
\hline \multicolumn{1}{c}{ Obreras Mayores } & \multicolumn{4}{c}{ Obreras Menores } \\
\hline & Prom. & D.E. & Max & Min & CV & Prom. & D.E. & Max & Min & CV \\
\hline LTC & 4,8 & 0,31 & 5,6 & 4,1 & 6,4 & 3,5 & 0,28 & 4,0 & 2,9 & 7,9 \\
AMC & 1,2 & 0,05 & 1,3 & 1,1 & 3,9 & 1,0 & 0,10 & 1,2 & 0,8 & 9,8 \\
LCC & 1,0 & 0,09 & 1,1 & 0,8 & 9,3 & 0,8 & 0,10 & 1,0 & 0,6 & 12,4 \\
AMP & 0,6 & 0,05 & 0,8 & 0,5 & 8,1 & 0,5 & 0,03 & 0,5 & 0,4 & 5,6 \\
LMP & 0,2 & 0,04 & 0,3 & 0,2 & 16,5 & 0,2 & 0,02 & 0,2 & 0,1 & 12,5 \\
LTP & 1,0 & 0,07 & 1,1 & 0,8 & 7,5 & 0,8 & 0,04 & 0,9 & 0,7 & 5,6 \\
\hline
\end{tabular}

Tabla 2. Correlaciones entre los caracteres morfométricos analizados en obreras de $C$. fulviceps. Referencias: cursiva: obreras mayores; normal: obreras menores; negrita y * indican correlaciones significativas $(\alpha \leq=0,05)$.

\begin{tabular}{ccccccc}
\hline & LTC & AMC & LCC & AMP & LMP & LTP \\
\hline LTC & 1 & 0,07 & $\mathbf{0 , 3 0 *}$ & 0,03 & $-0,12$ & 0,27 \\
AMC & 0,14 & 1 & $\mathbf{0 , 8 2} *$ & $\mathbf{0 , 3 7 *}$ & $\mathbf{0 , 4 2 *}$ & $\mathbf{0 , 4 8 *}$ \\
LCC & 0,01 & 0,11 & 1 & 0,15 & 0,25 & 0,28 \\
AMP & $\mathbf{0 , 6 1 *}$ & $\mathbf{0 , 4 8 *}$ & $-0,12$ & 1 & $\mathbf{0 , 3 4 *}$ & $\mathbf{0 , 3 6} *$ \\
LMP & $\mathbf{0 , 3 1 *}$ & $\mathbf{0 , 3 5 *}$ & $\mathbf{0 , 3 3 *}$ & $\mathbf{0 , 4 5 *}$ & 1 & 0,24 \\
LTP & 0,24 & $\mathbf{0 , 3 3 *}$ & 0,11 & $\mathbf{0 , 4 0 *}$ & 0,10 & 1 \\
\hline
\end{tabular}

Tabla 3. Análisis de la varianza de Kruskal-Wallis.

\begin{tabular}{ccccc}
\hline & \multicolumn{2}{c}{ Obreras $(\mathrm{gl}=1 ; \mathrm{n}=100)$} & \multicolumn{2}{c}{ Muestras $(\mathrm{gl}=4 ; \mathrm{n}=100)$} \\
\hline LTC & $\mathrm{H}$ & $\mathrm{p}$ & $\mathrm{H}$ & $\mathrm{p}$ \\
AMC & 74,30647 & 0,0000 & 8,819101 & 0,0658 \\
LCC & 50,68769 & 0,0000 & 4,772168 & 0,3115 \\
AMP & 69,33063 & 0,0000 & 15,09794 & 0,0045 \\
LMP & 63,59059 & 0,0000 & 0,9665574 & 0,9148 \\
LTP & 71,18910 & 0,0000 & 8,983047 & 0,0615 \\
\hline
\end{tabular}


Tabla 4. Análisis de componentes principales en caracteres morfométricos de obreras de $C$. fulviceps.

\begin{tabular}{cccccc}
\hline & Factor 1 & Factor 2 & Factor 3 & Factor 4 & Factor 5 \\
\hline Eigenvalues & 4.901 & 0.392 & 0.335 & 0.173 & 0.134 \\
\% Varianza Total & 81,68 & 6,54 & 5,59 & 2,88 & 2,23 \\
LTC & 0,922 & $-0,163$ & $-0,121$ & 0,317 & 0,026 \\
AMC & 0,940 & 0,146 & $-0,081$ & $-0,157$ & $-0,213$ \\
LCC & 0,858 & 0,490 & $-0,067$ & 0,085 & 0,048 \\
AMP & 0,929 & $-0,287$ & 0,028 & $-0,010$ & $-0,161$ \\
LMP & 0,857 & $-0,005$ & 0,504 & $-0,030$ & 0,092 \\
LTP & 0,912 & $-0,150$ & $-0,235$ & $-0,199$ & 0,225 \\
\hline
\end{tabular}

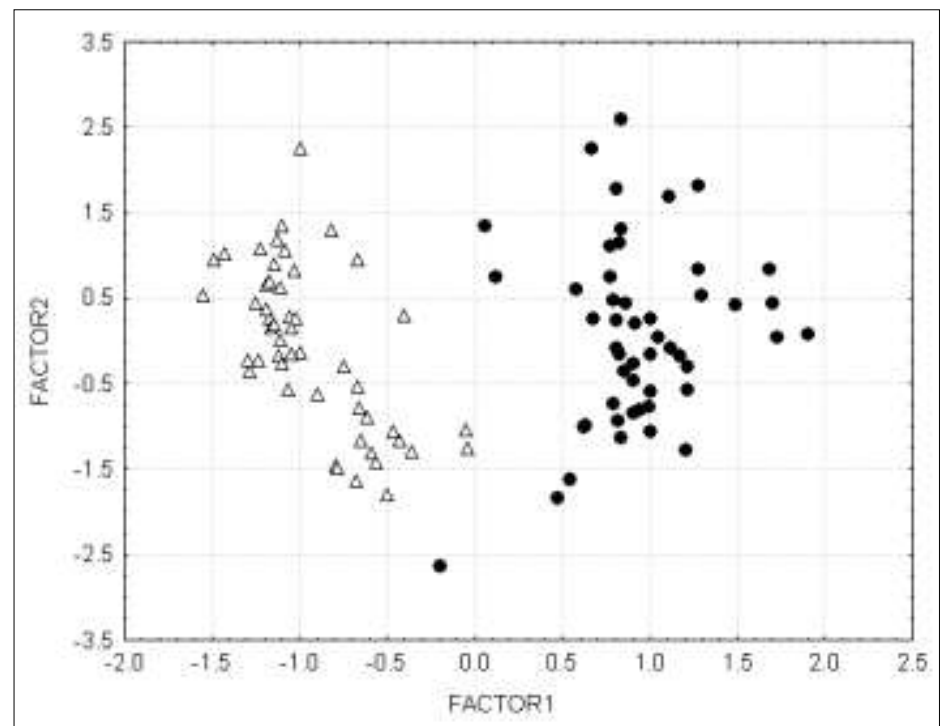

Figura 1. Posición de las obreras de C. fulviceps en los dos primeros factores extraídos en el análisis de componentes principales. Referencias: círculos negros = obreras mayores; triángulos blancos = obreras menores.

Realizando un gráfico de dispersión de las posiciones de las obreras sobre los dos primeros factores se observa la formación de dos grupos sobre el factor 1, lo cual confirma que este factor representa las diferencias de tamaño entre ambos grupos de obreras (Figura 1).

La existencia de obreras dimórficas es un rasgo común entre las Termitidae $\mathrm{y}$, de acuerdo al patrón de desarrollo que presenten, las diferencias observadas pueden deberse a dimorfismo sexual, donde las obreras menores son machos y las mayores hembras (Roisin, 2000), o representar diferentes estadíos del desarrollo (Noirot, 1969). En el género Nasutitermes, cercano a Cortaritermes, el dimorfismo entre las obreras se debe a la combinación de ambos factores, observándose al menos dos estadíos tanto en machos (obreras menores) como en hembras (obreras mayores) (Mc Mahan \& Watson, 1975; Buschini \& Costa Leonardo, 2002). En este trabajo no se determinó el sexo de las obreras analizadas, pero las diferencias observadas en el número de segmentos antenales entre obreras mayores y 
menores permiten suponer un patrón similar al observado en Nasutitermes, con al menos dos estadíos diferentes en cada tipo de obrera.

Por otra parte, las diferencias en los coeficientes de variaciones y correlaciones observadas en ambos grupos de obreras de Cortaritermes fulviceps indican que cada grupo presentan patrones de variabilidad diferentes, y que ese patrón se mantiene en las distintas colonias de una misma localidad, ya que no se encontraron diferencias significativas entre las muestras analizadas.

\section{CONCLUSIÓN}

Las obreras mayores y menores de $C$. fulviceps presentaron solapamiento en casi todos los caracteres morfométricos, las diferencias entre ambos grupos de obreras fueron significativas para todos las variables consideradas, cada grupo de obreras presentaron diferentes patrones de variabilidad siendo los caracteres del pronoto y de la tibia los más variables en las obreras mayores y las proporciones de la cabeza en las obreras menores.

\section{AGRADECIMIENTOS}

A la Secretaría General de Ciencia y Técnica de la Universidad Nacional del Nordeste por el financiamiento del proyecto F 015 "Bio-ecología de termites (Insecta, Isoptera) del Parque Nacional Mburucuyá (Corrientes, Argentina)" en el marco del cual se realizó este estudio, y a la Administración de Parques Nacionales por el apoyo brindado para el desarrollo de los muestreos de campo.

\section{REFERENCIAS BIBLIOGRÁFICAS}

BUSCHINI, M.L.T.; COSTA LEONARDO A.M.. 2002. Biometrics studies of caste development in Nasutitermes coxipoensis (Isoptera, Termitidae). Sociobiology, 40(2): $465-477$.

CONSTANTINO R. 1998. Catalog of the termites of the New World (Insecta:Isoptera). Arquivos de Zoología (Sao Paulo) 35(2): 135-230.

CORONEL, J.M.; PORCEL, E. 2001. Morphometric Variations in Soldiers of Termes saltans (Isoptera: Termitidae). Sociobiology 38 (3a): $465-474$.

CORONEL, J.M.; PORCEL, E.. 2002. Morphometric Analysis of Soldiers of Microcerotermes strunckii (Isoptera: Termitidae, Termitinae). Sociobiology 40 (2): $307-316$.

DARLINGTON, J.E.P.C.; ZIMMERMAN, P.R.; WANDIGA, S.O. 1992. Populations in nests of the termite Macrotermes jeanneli in Kenya. Journal of Tropical Ecology 8: 73 85.

HINZE, B.; CRAILSHEIM, K.; LEUTHOLD, R.H. 2002. Polyethism in food processing and social organisation in the nest of Macrotermes bellicosus (Isoptera, Termitidae). Insectes Sociaux 49: 31- 37.

HOSTETTLER, N.C.; HALL, D.W.; SCHEFFRAHN, R.H. 1995. Intracolony Morphometric Variation and Labral shape in Florida Reticulitermes (Isoptera: Rhinotermitidae) soldiers: Significance for identification. Florida Entomologist 78 (1): $119-129$.

MANZOOR, F.; AKHTAR, M.S. 2006. Morphometric Analysis Of Population Samples Of Soldier Caste Of Odontotermes parvidens Holmgren And Holmgren (ISOPTERA: Termitidae,Macrotermitinae). Journal of Research (Science), Bahauddin Zakariya University, Multan, Pakistan. 17 (3): 207-218. 
MC.MAHAN, E.A.; WATSON， J.A.L. 1975. Non-reproductives castes and their development in Nasutitermes exitiosus (Hill) (Isoptera). Insectes Sociaux. 22: $183-198$.

NOIROT, C. 1955. Recherches sur le polymorphisme des termites superieurs (Termitidae). Annales des Sciences Naturelles, Zoologie, 17: 399 - 595.

NOIROT, C. 1969. Formation of castes in the higher termites. In: Krishna, K. and F.M. Weesner (eds.) Biology of Termites. Vol. I. pp: $311-350$. Academic Press. New York.

ROISIN, Y. 2000. Diversity and evolution of caste patterns (p: 95 - 119). In: Abe, T., D.E. Bignell \& M. Matsumoto. (eds.) Termites: Evolution, Sociality, Symbiosis, Ecology. Kluwer Academic Publisher. 466 pp.

ROONWAL, M.L.; SANGAL, S.K. 1957. Variability in the mandibles of soldiers in the Odontotermes obesus (Rambur) (Isoptera, Family Termitidae). Records of the Indian Museum 55: 1 - 22. purposes. Journal of Zoological Society of India. 21(1): $9-66$.

THORNE, B.L. 1985. Numerical and biomass caste proportions in colonies of the termites Nasutitermes corniger and Nasutitermes ephratae (Isoptera, Termitidae). Insectes Sociaux 32 (4): $411-426$.

TORALES, G.J.; CORONEL, J.M.; GODOY, M.C.; LAFFONT, E.R.; ROMERO, V.L. 2008. Additions to the Taxonomy and Distribución of Isoptera From Argentina. Sociobiology 51 (1): $31-47$.

TORALES, G.J.; LAFFONT, E.R.; GODOY, M.C.; CORONEL, J.M.; ARBINO, M.O. 2005. Update on Taxonomy and Distribution of Isoptera from Argentina. Sociobiology 45 (3): $853-886$.

ROONWAL, M.L. 1969. Measurements of termites (Isoptera) for taxonomic 